\title{
Home-Based Monitoring of Pulmonary Function in Patients with Duchenne Muscular Dystrophy
}

\author{
Gunnar M. Buyse ${ }^{\mathrm{a}, *}$, Christian Rummey ${ }^{\mathrm{b}}$, Thomas Meier ${ }^{\mathrm{c}}$, Mika Leinonen ${ }^{\mathrm{c}}$, \\ Thomas Voit ${ }^{\mathrm{d}}$, Craig M. McDonald ${ }^{\mathrm{e}}$ and Oscar H. Mayer ${ }^{\mathrm{f}}$ for the DELOS Study Group \\ ${ }^{a}$ University Hospitals Leuven, Leuven, Belgium \\ ${ }^{\mathrm{b}}$ Clinical Data Science, Basel, Switzerland \\ ${ }^{\mathrm{c}}$ Santhera Pharmaceuticals, Pratteln, Switzerland \\ ${ }^{\mathrm{d}}$ UCL Great Ormond Street Institute of Child Health, NIHR Great Ormond Street Hospital \\ Biomedical Research Centre, London, UK \\ ${ }^{\mathrm{e}}$ University of California Davis Medical Center, Sacramento, USA \\ ${ }^{\mathrm{f}}$ The Children's Hospital of Philadelphia, Philadelphia, USA
}

\begin{abstract}
.
Background: Loss of pulmonary function is a main cause of early morbidity and mortality in patients with Duchenne muscular dystrophy (DMD). Standard of care guidelines recommend regular assessment of pulmonary function by hospital-based spirometry to detect onset and monitor progression of pulmonary function decline.

Objective: To assess the feasibility of home-based monitoring of pulmonary function by a hand-held device (HHD) in adolescent and adult patients with DMD over a period of 12 months.

Methods: In the phase III randomized placebo-controlled DELOS trial in 10-18 year old DMD patients, peak expiratory flow (PEF) measurements were collected weekly at home by the patient (assisted by parent/caregiver) using a peak flow meter HHD. Adherence to the use of the HHD was assessed and 12-month changes in PEF as percent of predicted (PEF\%p) for the idebenone $(\mathrm{N}=31)$ and the placebo treatment groups $(\mathrm{N}=33)$ from HHD-derived data were compared to results from hospital-based spirometry.

Results: A total of 2689 individual HHD assessments were analysed. Overall adherence to the use of the HHD over the course of the 12-month study duration was good (75.9\%, SD 21.5\%) and PEF\%p data obtained at the same day by HHD and standard spirometry correlated well (Spearman's rho $0.80 ; p<0.001$ ). Several analysis methods of HHD-derived data for PEF\%p consistently demonstrate that idebenone treatment slowed the decline in PEF\%p compared to placebo, which supports the statistically significant difference in favour of idebenone for PEF\%p measured by standard spirometry.

Conclusions: This study demonstrates that home-based monitoring of pulmonary function in adolescent patients with DMD using a HHD is feasible, provides reliable data compared to hospital-based spirometry and is therefore suitable for use in clinical practice and for clinical trials.
\end{abstract}

Keywords: Pulmonary function, respiration, idebenone, Duchenne muscular dystrophy, peak expiratory flow

\footnotetext{
*Correspondence to: Professor Dr Gunnar M. Buyse, MD, PhD, Paediatric Neurology, University Hospitals Leuven, Herestraat 49,
} 


\section{INTRODUCTION}

Duchenne muscular dystrophy (DMD) is usually diagnosed at age 3-5 years when children start to show signs of muscle weakness. Loss of ambulation is the first irreversible disease milestone, typically occurring during early teenage years. Coinciding with loss of ambulation, pulmonary function starts to decline and ultimately progresses to the stage of chronic respiratory failure with patients requiring assisted ventilation, initially nocturnal and then diurnal and finally continuously. Becoming dependent on assisted ventilation represents a key irreversible disease milestone for patients with DMD, occurring years after loss of ambulation. Despite major progress in patient care including ventilator support [1], cardio-pulmonary complications remain the major cause of morbidity and early mortality in DMD [2-6].

Clinical complications from respiratory muscle weakness include inability to cough efficiently, leading to poor airway clearance and mucus plugging of airways, recurrent respiratory infections, which frequently require hospitalization, and respiratory failure resulting in the need of ventilator support [6]. DMD patients and their caregivers consider the preservation of pulmonary function, particularly maintaining effective cough and reducing the risk of airway infections, important treatment goals [7]. Safeguarding pulmonary function, therefore, represents a significant unmet need.

As emphasized by several standard of care recommendations and guidelines [2, 6, 8-10] serial assessment of pulmonary function is a critical element of routine monitoring for patients with DMD, especially when they become non-ambulant. The most frequently used pulmonary function parameters across all age ranges are the volume-related forced-vital capacity (FVC) and the flow-related peak expiratory flow (PEF), typically measured by spirometry during hospital visits. To account for maturational changes, pulmonary function outcomes are normalized to patient age, height, gender and race and expressed as percent of predicted (PEF\%p, FVC\%p). Ulna length is routinely used in clinical practice to derive a surrogate measure of patient height for normalization of pulmonary function testing results [11-13], particularly in patients who have become non-ambulant, have developed joint contractures and are unable to stand upright. Although effort-dependent, FVC\%p and PEF\%p can reliably be measured in school age patients as shown by low within-subject coefficients of variation (CV) for successive measures [14]. Several studies have independently demonstrated that PEF\%p and FVC\%p follow a co-linear decline between the age of around 10-20 years [15-18]. Recent studies also indicate that PEF\%p might be a more sensitive measure of early decline in pulmonary function since PEF\% pegins to decline before FVC\%p [16, 18-20] and in contrast to $\mathrm{FVC} \% \mathrm{p}$ decline in PEF\% $\mathrm{p}$ can be seen already in ambulant patients [20].

Standard of care guidelines recommend regular monitoring of pulmonary function and command change in patient care when decline in pulmonary function drops below defined thresholds $[2,6,11,17]$. However, as recently demonstrated in a study with 770 DMD patients from Europe and the US, compliance for routine pulmonary function monitoring is generally poor [21]. Specifically, this study found that depending on country only in $61-81 \%$ of patients the guideline of 6-monthly pulmonary function monitoring was fulfilled.

Therefore, there is a need for a more versatile, ideally home-based approach to obtain pulmonary function data from patients with DMD. Such data, if collected regularly, could provide treating physicians an indicator of the rate of pulmonary function decline and help identify patients at risk of falling below clinically relevant thresholds of pulmonary function, which would then indicate the need for a change in clinical management. In addition, more frequent home-based assessments of pulmonary function may offer statistical advantages for the evaluation of treatment effects in clinical trials.

In this study we evaluated the use of a versatile, child-compatible hand-held device (HHD) that DMD patients could apply for pulmonary function testing at home. We hypothesized that home-based spirometry is feasible as defined by adherence to the procedure, and we further hypothesized that weekly home spirometry would produce comparable trends to data collected by in-clinic spirometry testing.

\section{METHODS}

\section{Clinical trial conduct}

The randomized, placebo-controlled, Phase III DELOS study of idebenone in patients with DMD was conducted between July 2009 and December 2013 in study centres located in Belgium, Germany, the Netherlands, Switzerland, France, Sweden, Austria, Italy, Spain, and the USA. The trial and any 
changes to the protocol were approved by relevant national authorities and the institutional review boards or independent ethics committees in the countries of the participating centers and conducted in accordance with good clinical practice and the principles of the Declaration of Helsinki. Prior to any study related procedure, written informed consent was obtained from all patients and/or parents or guardian. The DELOS trial (clinicaltrials.gov ID: NCT01027884) had the objective to assess the efficacy of idebenone, compared to placebo, in improving or delaying the loss of respiratory function in patients with DMD. The study design and overall results have been previously published [22]. The study met its primary endpoint and demonstrated a statistically significant and clinically relevant reduction of pulmonary function decline, as measured by the primary endpoint, change to week 52 in PEF\%p, using in-clinic spirometry testing. Further work reported analyses of the efficacy of idebenone treatment on respiratory complications [23, 24] and inspiratory function [25] as well as a detailed characterisation of the baseline status of enrolled patients and the progression of pulmonary function loss in the placebo group [14].

\section{Methods of pulmonary function testing}

To ensure the collection of reliable pulmonary function data, the DELOS study protocol only allowed enrolment of patients who were able to perform a tight mouth seal. In addition, patients had to provide reproducible PEF\%p results between screening and baseline (completed within 6 weeks after screening) with a variability of less than $15 \%$ in PEF\%p. The observed coefficient of variation between screening and baseline was $6.97 \%$ for PEF\% p which was comparable to a coefficient of variation of $6.69 \%$ for FVC\%p [14]. Assessment of PEF\%p was performed by two independent methods at different intervals and frequency. The primary analysis of changes in PEF\%p was based on inclinic spirometry conducted at baseline and weeks 13, 26, 39 and 52 using a Pneumotrac Spirometer 6800 (Vitalograph). Independently, PEF\% p data were also collected weekly at home with the portable, child compatible electronic pulmonary monitoring device (asma-1/nmd-1; usb model 4000; Vitalograph) which was used with a disposable tube-shaped cardboard mouthpiece (Fig. 1).

This hand-held device (HHD) is a validated, battery powered, portable electronic peak flow meter for monitoring pulmonary function applying a stator/rotor mass flow principle to measure PEF. The flow range for PEF is $25-840 \mathrm{~L} / \mathrm{min}$, device accuracy is better than $+/-10 \%$ or $+/-25 \mathrm{~L} / \mathrm{min}$, whichever is greater and device repeatability is better than $5 \%$ or $10 \mathrm{~L} / \mathrm{min}$, whichever is greater. This HHD is CEmarked in accordance with the EU Medical Devices Directive 2007/47/EC, has FDA 510k registration and

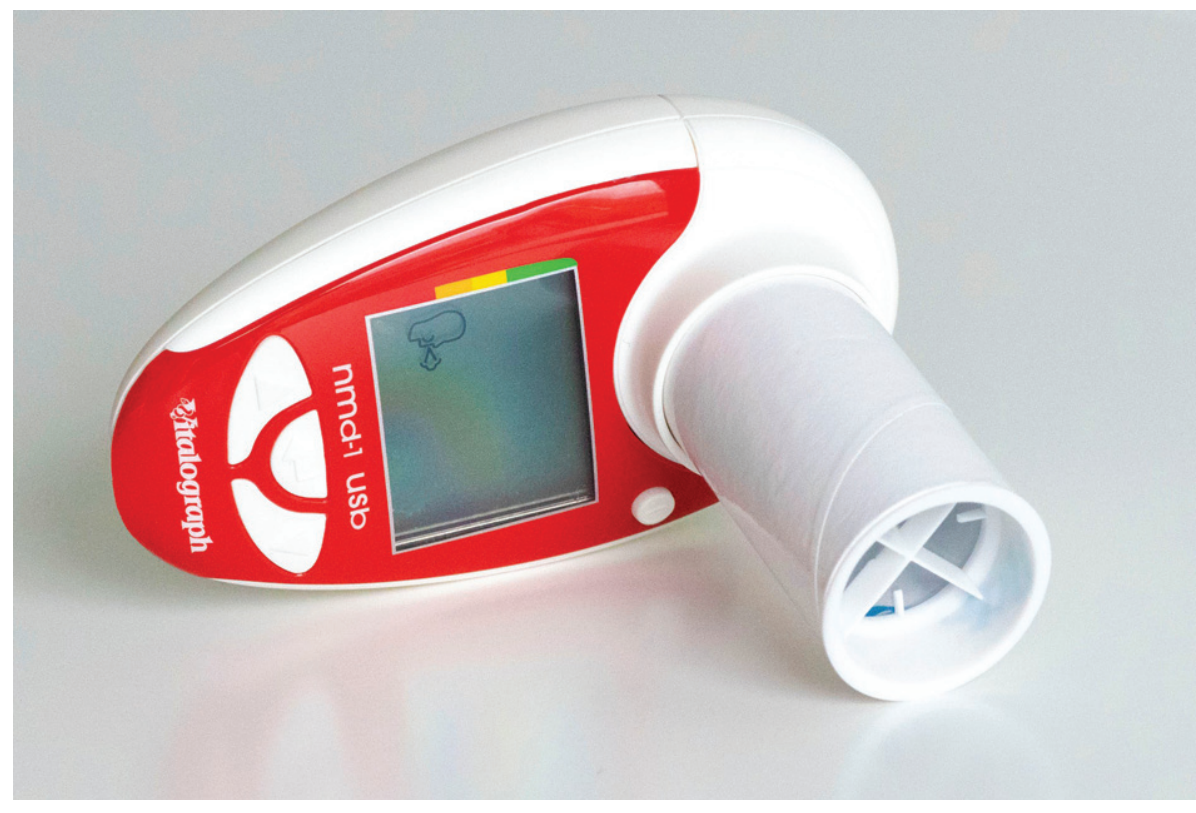

Fig. 1. Hand-held device (HHD) and mouthpiece used in this study. 
was tested to meet accuracy and precision as specified by the American Thoracic Society (ATS) and European Respiratory Society (ERS) 2005 recommendations [26]. The device precludes unintentional manipulation of stored data.

\section{Collection of pulmonary function data}

At each hospital visit of the DELOS study the assessment of PEF was the first pulmonary function test to be conducted by spirometry. For the PEF-manoeuvre, study participants were instructed to exhale all of the air out of the lung as fast as possible, until the lungs are completely empty. The highest available test result of PEF (in L/min) was used for further analysis and PEF\%p calculated centrally by using patient ulna length data to derive patient height [22].

At the study start patients and caregivers were instructed and trained by a physician, physical therapist or study nurse on the use of the HHD, which was to be conducted once per week at home, preferentially on a specified day. Patients received monthly phone calls from the study co-ordinator to remind them to perform the tests with the HHD. In addition to the home-based PEF assessments, patients were asked to perform pulmonary function test with the HHD at each hospital visit following the spirometry test. During each study site visit, all data recorded on the HHD were downloaded via USB connection and transferred to the study database.

\section{Normalization of pulmonary function data to percent or predicted (\%p)}

For each pulmonary function test (either in-clinic spirometry or with HHD), the highest value from a minimum of three and up to five consecutive manoeuvres was used for analysis. To calculate PEF\%p, raw data of PEF (measured in L/min) were normalized for height (derived from ulnar length $[12,13]$, using established conversion equations [27] (for details see [22]).

\section{Data analyses}

Analyses were conducted in the ITT population, except for the linear regression analysis (Fig. 2: Method 1), where 4 patients did not provide data for up to at least Month 6 and for the MMRM analysis of all weekly data (Fig. 2, Method 4), where 6 patients did not provide a baseline value). Individual adherence to the device was assessed by calculating the proportion of weeks that a patient provided HHD data divided by the number of study weeks with exposure to study medication. For subjects who discontinued the study, HHD data was used up to the day of last medication intake. Results from the HHD device (individual measurements) and the progression over 52 weeks were also compared graphically with the results from spirometry data collected in 3monthly intervals during clinic visits. To assess how well the individual regression lines fitted the HDD data, we calculated the mean percental residuals over

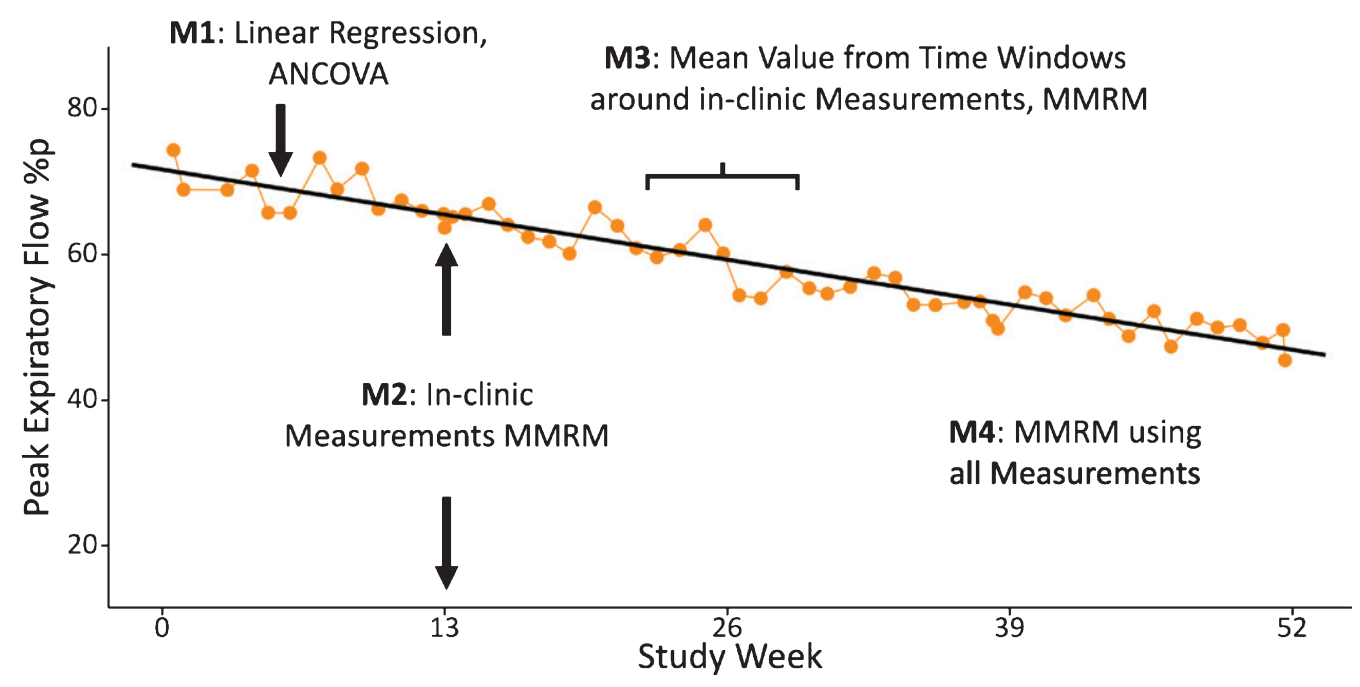

Fig. 2. Illustration of four methods (M1 to M4) applied to analyse HDD data. 
all data points per subject and categorized this value into up to $5 \%$, between $5 \%$ and $10 \%$ and over $10 \%$. For a subject in the $<5 \%$ category, the mean deviation of all measurements from the regression line was less than $<5 \%$.

Data from the HHD was analysed using four different statistical methods (see Fig. 2).

Method 1: For the pre-defined primary analysis of HDD data in the DELOS trial, linear regression slopes were fitted for every individual subject, using all available weekly HHD measurements. The individual slopes were then analysed with an ANCOVA model using the intercept of the regression as Baseline covariate.

Method 2: HDD values obtained from the in-clinic measurements were analysed with a mixed model for repeated measures (MMRM), using the baseline value as covariate. The main purpose of this analysis was to allow direct comparison between HHDderived data and hospital spirometry data obtained at the same day during study site visit.

Method 3: For this window-based approach an analogous MMRM analysis was performed similar to Method 2. However, for this method, the mean values from all HHD data available from the 3-weeks before and after the study site visits was calculated. For the visits at study start only the 3 weeks following the baseline visit were included and for the week 52 visit only the 3-weeks prior to this last study visit were used. Sensitivity analyses using a similar approach were conducted using 2-week and 4-week windows.

Method 4: A repeated measures MMRM analysis was conducted using all weekly data collected with the HHD. In this case study week was used as fixed factor.

The primary endpoint in the DELOS trial was the difference between treatment groups for the change in PEF \%p from Baseline to the week 52-time point, assessed by an MMRM using the treatment group contrast at the week 52-time point [22]. The primary method for analysing HHD data (linear regression) assesses the overall progression and separation of the treatment groups during the study period, not restricted to the week 52-time point. For an appropriate comparison, for Methods 2-4 also the overall study treatment differences were calculated (assessed by the overall study treatment group contrast).

Statistical analyses were conducted using R 3.4.4 [28] and SAS 9.3 (SAS Institute Inc., Cary, North Carolina, USA).

\section{RESULTS}

Patients

Detailed demographic characteristics and baseline pulmonary function of the DELOS study population were previously published [14, 22]. Briefly, patients with DMD enrolled in the DELOS trial were between 10 and 18 years of age (mean: 14 years) and were required to be off glucocorticoids $(\mathrm{GC})$ for at least 1 year prior to enrolment and GC use was excluded during the 52-week study period. Patients with asthma, bronchitis/COPD, bronchiectasis, emphysema, pneumonia or the presence of any other non-DMD respiratory illness that affects pulmonary function testing were also excluded (see [22] for details on inclusion/exclusion criteria). All patients in the study were able to perform the pulmonary function testing with the HHD. Study participants had to be in the pulmonary function decline stage, assessed as PEF\%p of $\leq 80 \%$ at screening (and confirmed at baseline). Patients enrolled into DELOS presented with advanced stage of the disease as assessed by upper and lower extremity weakness: Brooke upper extremity score was $4.2 \pm 1.2$ (mean, SD) with $59.4 \%$ of patients unable to raise their hand to the mouth (Brooke score 5 or 6 ); $92.2 \%$ of study participants were non-ambulant at baseline [14]. In total, 64 patients were randomized, 31 patients received idebenone (Raxone ${ }^{\circledR} 150 \mathrm{mg}$ tablets, Santhera Pharmaceuticals) at a daily dose of $900 \mathrm{mg}$ (given as 3 times $300 \mathrm{mg}$ taken with meals) and 33 received placebo. Randomization was stratified according to baseline PEF\%p value (PEF\%p $<40 \%$ or 40 to $80 \%$ ).

\section{Adherence to the HHD}

Adherence to the use of the HHD was assessed by calculating the number of weeks a patient provided HDD data divided by the number of weeks of exposure to study medication (Fig. 3). Overall mean adherence in the use of the HHD was calculated by weighted mean of individual adherences, as summarised in Table 1.

During this study a total of 2689 PEF-measures (counting only the highest available PEF measure from each daily test) were collected. Overall adherence to the weekly use of the HHD was very good with over $75 \%$ overall, which suggests that on average a patient missed 8 weekly measurements during the 52week study period. Only 8 patients (4 on idebenone 


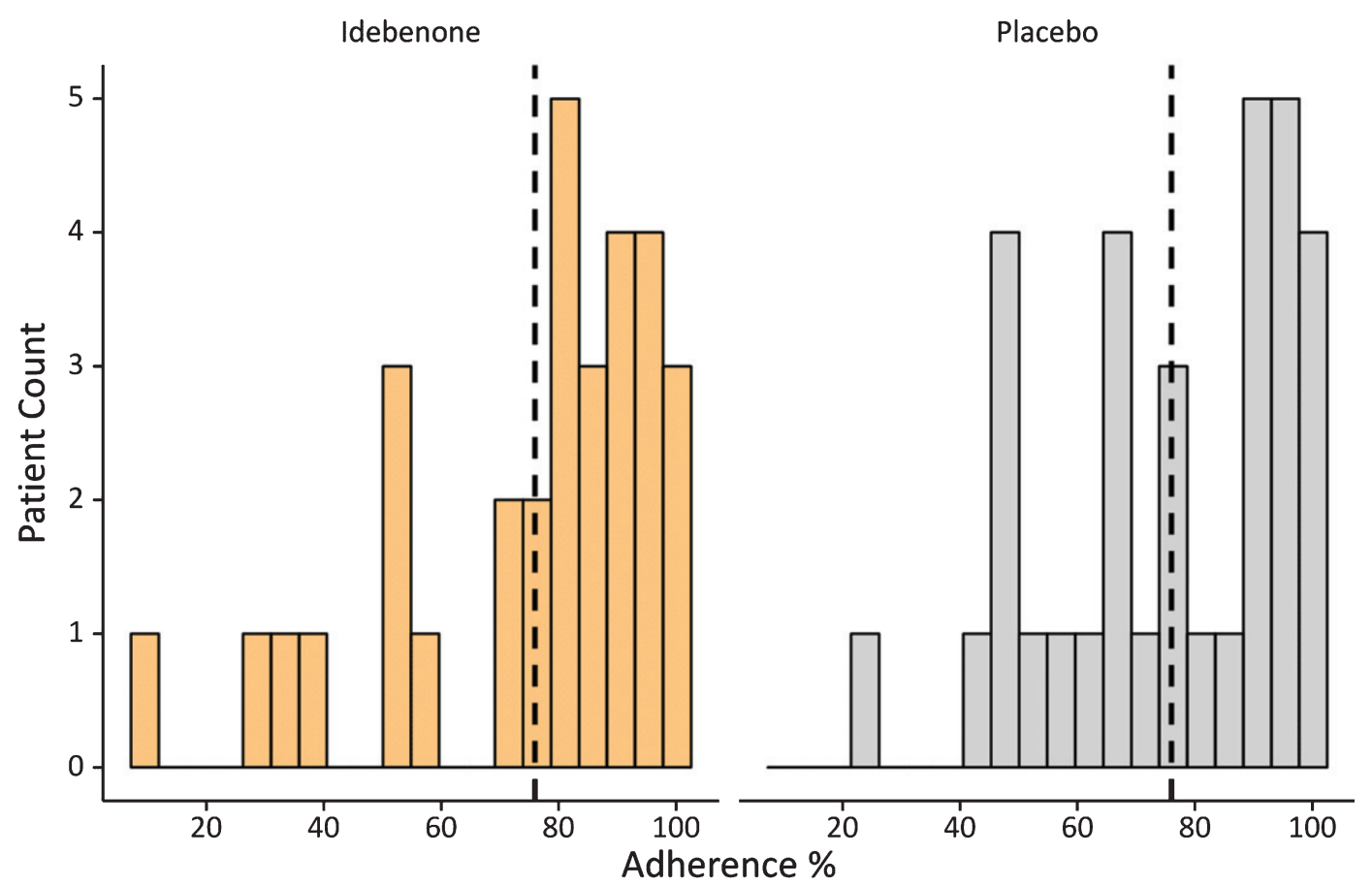

Fig. 3. Distribution of adherence to HHD pulmonary function testing. Vertical lines indicate overall median adherence value.

Table 1

Adherence to pulmonary function assessment using the HHD device

\begin{tabular}{lc}
\hline Group & Adherence to HHD use* \\
\hline Idebenone $(n=31)$ & $76.3 \%$ (SD 22.0) \\
Placebo $(n=33)$ & $75.5 \%$ (SD 21.1) \\
Total $(n=64)$ & $75.9 \%$ (SD 21.5) \\
\hline
\end{tabular}

*Data is weighted mean (Standard Deviation, SD) of percentage of weeks in study with at least one home-based measurement.

and 4 on placebo) had an adherence of less than $50 \%$ (Fig. 3). Variability in the adherence of HHD use was study-site specific: whilst overall compliance was well above $75 \%$ (in centres with more than 3 patients), there was one study site with five patients with a mean adherence of $35 \%$, which might have resulted from inappropriate instructions provided by study site personnel to participants. In addition, we assessed adherence on a weekly and quarterly basis over the course of the 52-week study period (Fig. 4).

Adherence was around $80 \%$ in the first quarter in both treatment groups, and remained relatively stable at approximately $75 \%$ in the idebenone group throughout the remaining study period. In the placebo group, adherence dropped to $71 \%$ in the third quarter and eventually to $67 \%$ in the fourth quarter of the study (Fig. 4). We tested whether the slightly lower and declining adherence in the placebo group could have been a consequence of the declining pulmonary function and resulting discouragement of study participants from regularly conducting the home-based testing. A correlation analysis (not shown) did not reveal reduced adherence in patients with declining pulmonary function. In summary, these data indicate that patients were generally comfortable using this HHD during the 52-week study period and the overall adherence was good.

\section{Comparison of pulmonary function results obtained by HHD with in-clinic spirometry results and intra-subject variability}

PEF\%p data obtained with the HHD from exemplary individuals are shown in Fig. 5 and compared to results obtained by spirometry during study visits at baseline and weeks 13, 26, 39 and 52. From this visual inspection of all available data obtained by inclinic spirometry and HHD per subject (as shown in Fig. 5) it can be concluded that data obtained by HHD match very well the data obtained by hospital spirometry. For the majority of patients $(41,64 \%)$ the mean percental residuals were less than $5 \%$ (examples in Fig. 5A). For another 21 (33\%) it was between 5\% and $10 \%$ (Fig. 5B) and only for 2 patients (3\%) the mean 


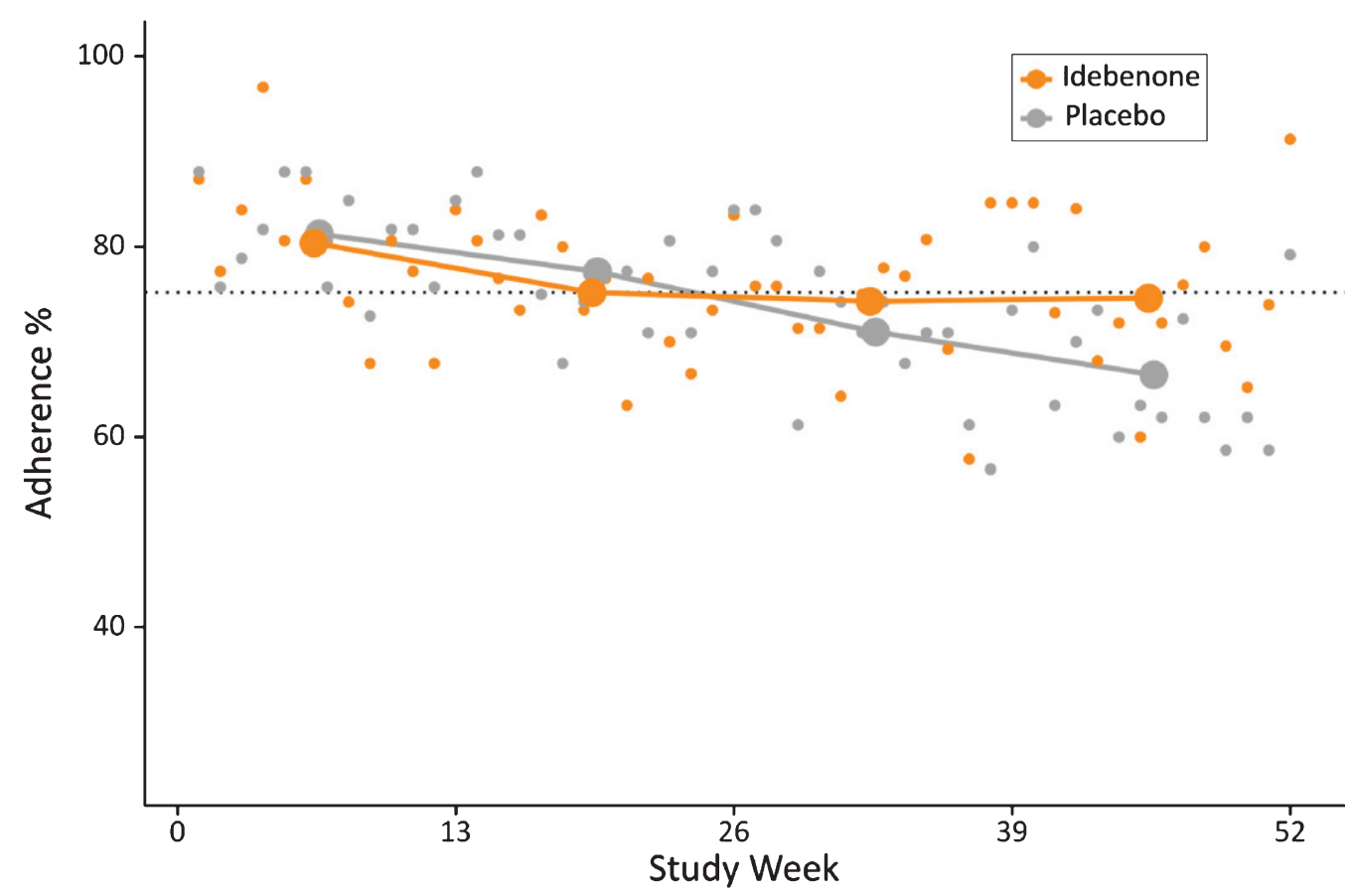

Fig. 4. Weekly and quarterly adherence to the HDD pulmonary function testing during the 52-week study period. Small dots indicate weekly average adherence, and connected large dots show mean quarterly adherence by treatment group. The dotted line shows overall mean compliance $(75.9 \%)$.

of percental residuals was larger than $10 \%$ (Fig. 5C). For these two patients the HDD data suggests that in certain phases of the study the home-based measurements deviated from hospital-based spirometry data.

To further assess the comparability of PEF\%p data obtained by spirometry and by HHD, data obtained with both methods at the same day during hospital visits at Baseline and week 13, 26, 39 and 52 were correlated. The resulting Spearman's rho correlations were $0.89(p<0.001)$ for PEF $[\mathrm{L} / \mathrm{min}]$ and $0.80(p<0.001)$ for PEF\%p, indicating overall very good data correlation between spirometry and HHD assessments.

Comparative analysis between spirometry-and $H H D$-assessed data for the treatment effect of idebenone on PEF\%p

The DELOS study investigated the effect of idebenone compared to placebo for the change in PEF\%p from baseline to week 52. The primary endpoint was the difference between treatment groups for hospital spirometry measured PEF\%p and calculated by a mixed model for repeated measures. As described previously [22], the rate of decline for
PEF\%p was higher (-8.84\%p) in the placebo group compared to the idebenone group $(-2.57 \% \mathrm{p})$, resulting in a statistically significant between-treatment group difference in favour of idebenone of $6.27 \% \mathrm{p}$ at week 52 and of $6.52 \%$ p when all post-baseline visits were used for the calculation (Table 2).

When these data are compared to results obtained with the HHD at the hospital visits (Method 2), the observed between-treatment group difference in favour of idebenone for PEF\%p was $9.73 \% \mathrm{p}$ for the change to week 52 and $7.48 \%$ p for the analysis including all post-baseline visits. These outcomes are in the same range but numerically larger than the treatment difference observed by hospital spirometry. From additional methods used to analyse HHD-derived data (regression analysis, window-based approach and all individual weekly assessments, Method 1, 3 and 4) it appears that linear regression analysis matches best the results obtained by hospital-based spirometry outcomes. The window-based analysis approach (using a 3-week window) reasonably matched the spirometry analysis at week 52 but underestimated the treatment effect of idebenone when all post-baseline data are included. Using the analysis with all individual post-baseline data (Method 4), also slightly underestimated the 

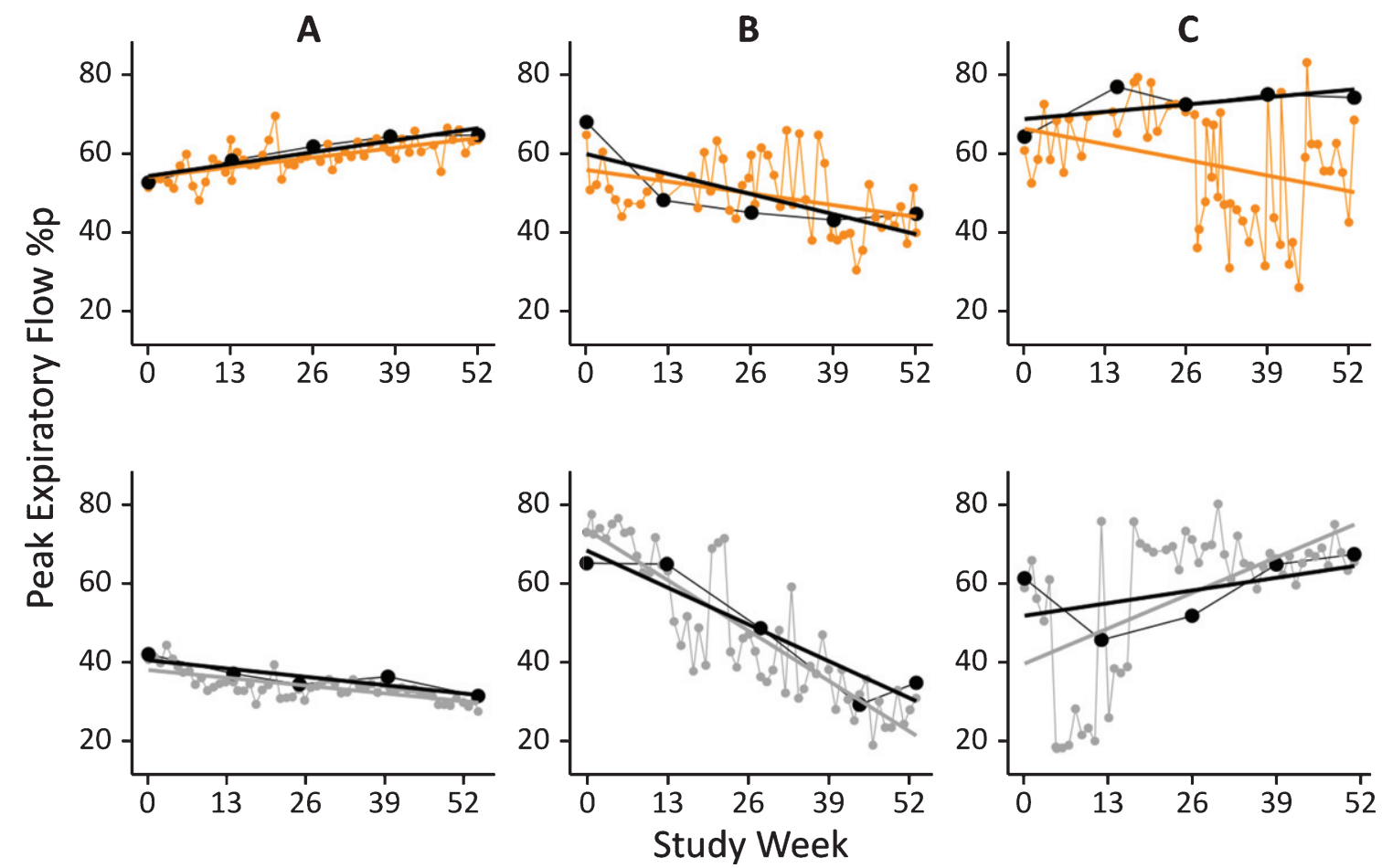

Fig. 5. PEF\% data obtained by HHD from 6 representative patients. Weekly HHD data are shown as coloured dots (orange, top row: patients receiving idebenone, grey, bottom row: patients receiving placebo). Linear regression lines derived from HHD data (colored line), and derived from hospital based spirometry data (black dots and black line) are shown for comparison.

idebenone treatment effect compared to in-clinic spirometry results.

A graphical by-patient comparison for the change in PEF\%p from baseline to week 52 between inclinic spirometry results and HHD data analysed by linear regression analysis (Method 1) is shown in Fig. 6. In general the 12-month changes in PEF\%p assessed by these methods match very well. There were only 8 patients for whom the change in $\mathrm{PEF} \% \mathrm{p}$ over the 12-month study period deviated by more than $20 \%$ between in-clinic spirometry and HHD-assessed results.

We also performed sensitivity analyses for the window-based approach by applying differing width of the analysis window around hospital visits $(2,3$, or 4 -week windows before and after a hospital visit). As shown in Table 3, comparable results were obtained, indicating that a short observation of several weeks already provides a reliable assessment of the changes in PEF\%p.

Trajectories for the change from baseline in $\mathrm{PEF} \% \mathrm{p}$ for each treatment group were comparable between the spirometry-based measures and those obtained weekly at home using the HHD (Fig. 7).
Although mean HHD-data show variability from week to week, their change by treatment group is well in line to what is recorded by hospital spirometry, resulting in clearly diverging trajectories for PEF\%p for the idebenone and placebo treatment groups for the study period. Of particular relevance is the observation that $95 \%$ confidence intervals from data obtained with spirometry and those obtained by the HHD are overlapping, demonstrating that both assessment methods for PEF\% $\mathrm{p}$ were congruent and mutually supportive.

\section{DISCUSSION}

This study is the first report on the feasibility of a hand-held device for frequent home-based monitoring of pulmonary function in patients with DMD. Our data demonstrate that the weekly use of a HHD was well accepted by adolescent trial participants over the course of 1 year, with average compliance exceeding $75 \%$, which compares well to a recent study with HHD use in adult patients with COPD [29]. The findings of this study have implications on 
Table 2

Comparative analyses for spirometry- and HHD-assessed outcomes for the effect of idebenone on the change PEF\%p

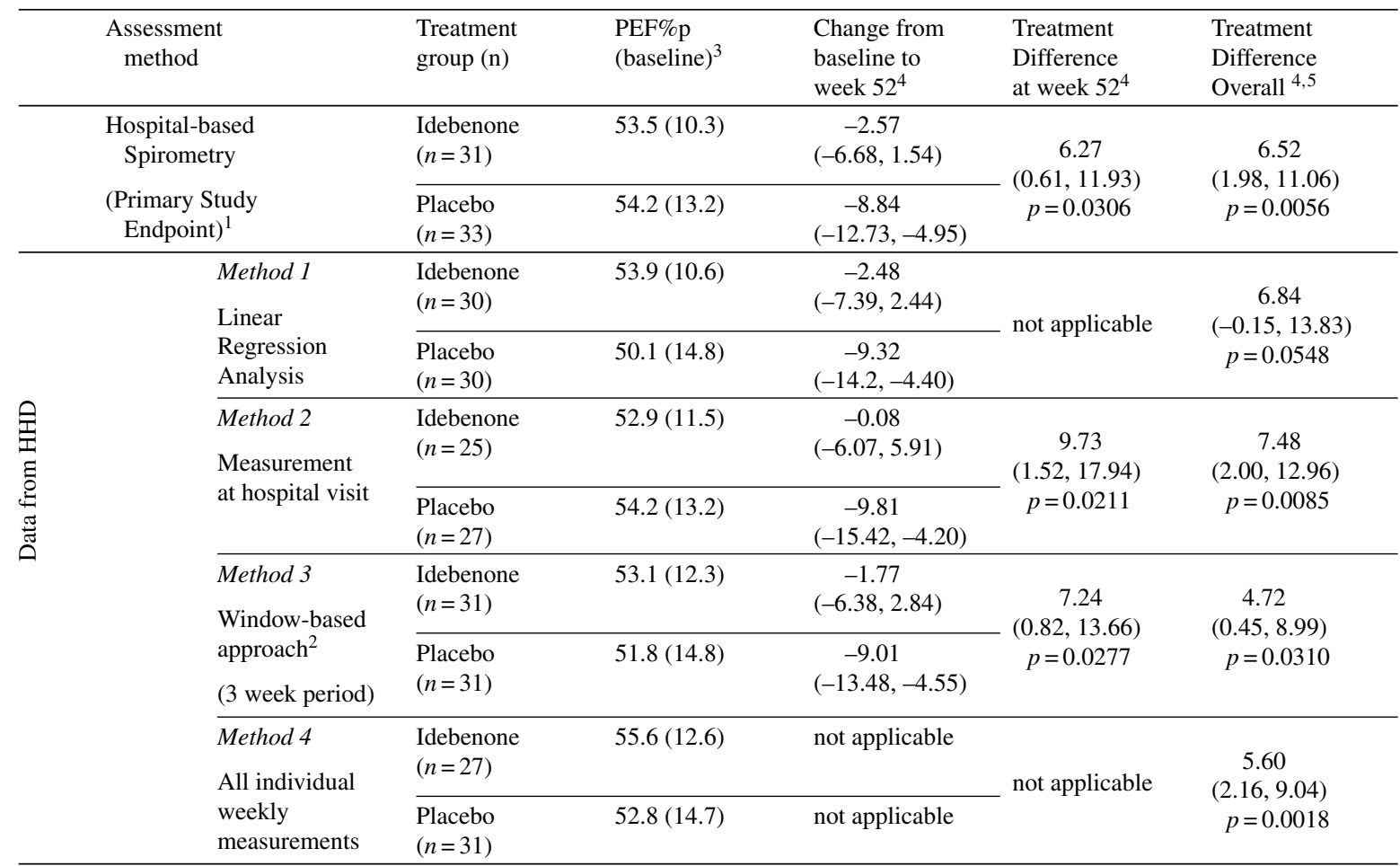

Method 1-4: analyses methods as described in Fig. 2. 1: as reported in (Buyse et al., 2015); 2: data averaged from a time window \pm 3 weeks around hospital visits; 3: data is mean (SD); 4: data is estimated mean from MMRM (95\% CI); 5: for the treatment difference overall, all post-baseline values were included in the model.

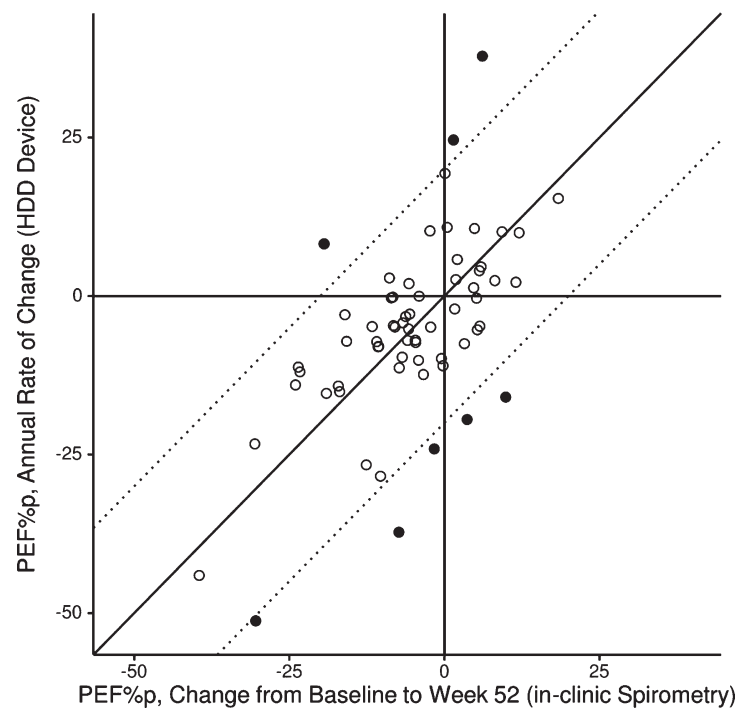

Fig. 6. Scatterplot for individual patients $(\mathrm{N}=64)$ for change in PEF\%p from baseline to week 52 by assessment method. Diagonal line: indicates perfect match; dashed lines: deviation of $20 \%$ between methods; filled circles: 8 patients for whom the change in PEF\% over the 12-month study period deviated more than $20 \%$ between assessment methods. the planning of future clinical trials aimed to assess the therapeutic potential of drug candidates on pulmonary function outcomes in patients with DMD. As demonstrated here, results obtained by hospital spirometry for the primary outcome of the DELOS trial, the change in PEF\%p, were independently supported by results obtained with the HHD. Although numerical differences for spirometry-measured and HHD-measured outcomes are observed, the point estimates for the between-group differences are of similar magnitude and consistently demonstrated a treatment effect in favour of idebenone in preserving PEF\%p compared to placebo. Despite the limited sample size of 31 and 33 patients per treatment group, this consistency of findings across different analysis methods for the HHD-derived data is encouraging and independently supports the positive result for the primary endpoint of the DELOS trial demonstrating that idebenone slowed pulmonary function loss in patients with DMD. Similar approaches whereby HHD-derived pulmonary function data were used to support clinical trial findings obtained by hospital spirometry were previously reported for more 
Table 3

Influence of the window size on outcomes for change in PEF\% by treatment group

\begin{tabular}{|c|c|c|c|c|c|}
\hline Window Size & $\begin{array}{l}\text { Treatment } \\
\text { group }(\mathrm{n})\end{array}$ & $\begin{array}{l}\text { PEF\%p } \\
\text { (baseline) }^{1}\end{array}$ & $\begin{array}{l}\text { Change from } \\
\text { baseline } \\
\text { to week } 52^{2}\end{array}$ & $\begin{array}{l}\text { Treatment } \\
\text { Difference } \\
\text { at week } 52^{2}\end{array}$ & $\begin{array}{l}\text { Treatment } \\
\text { Difference } \\
\text { Overall }^{2,3}\end{array}$ \\
\hline \multirow{2}{*}{2 weeks } & $\begin{array}{l}\text { Idebenone } \\
(n=31)\end{array}$ & $53.1(12.3)$ & $\begin{array}{c}-1.95 \\
(-6.56,2.66)\end{array}$ & \multirow{2}{*}{$\begin{array}{c}7.73 \\
(1.30,14.16) \\
p=0.0192\end{array}$} & \multirow{2}{*}{$\begin{array}{c}5.19 \\
(0.80,9.58) \\
p=0.0212\end{array}$} \\
\hline & $\begin{array}{l}\text { Placebo } \\
(n=31)\end{array}$ & $52.5(14.9)$ & $\begin{array}{c}-9.68 \\
(-14.15,-5.20)\end{array}$ & & \\
\hline \multirow{2}{*}{$\begin{array}{l}3 \text { weeks } \\
\text { (main analysis) }\end{array}$} & $\begin{array}{l}\text { Idebenone } \\
(n=31)\end{array}$ & $53.1(12.3)$ & $\begin{array}{c}-1.77 \\
(-6.38,2.84)\end{array}$ & \multirow{2}{*}{$\begin{array}{c}7.24 \\
(0.82,13.66) \\
p=0.0277\end{array}$} & \multirow{2}{*}{$\begin{array}{c}4.72 \\
(0.45,8.99) \\
p=0.0310\end{array}$} \\
\hline & $\begin{array}{l}\text { Placebo } \\
(n=31)\end{array}$ & $51.8(14.8)$ & $\begin{array}{l}-9.01 \\
(-13.48,-4.55)\end{array}$ & & \\
\hline \multirow{2}{*}{4 weeks } & $\begin{array}{l}\text { Idebenone } \\
(n=31)\end{array}$ & $52.8(12.6)$ & $\begin{array}{c}-1.39 \\
(-6.10,3.32)\end{array}$ & \multirow{2}{*}{$\begin{array}{c}7.63 \\
(1.06,14.21) \\
p=0.0236\end{array}$} & \multirow{2}{*}{$\begin{array}{c}4.98 \\
(0.65,9.30) \\
p=0.0248\end{array}$} \\
\hline & $\begin{array}{l}\text { Placebo } \\
(n=31)\end{array}$ & 51.7 (14.6) & $\begin{array}{c}-9.03 \\
(-13.61,-4.44)\end{array}$ & & \\
\hline
\end{tabular}

1: data is mean (SD); 2: data is estimated mean from MMRM $(95 \% \mathrm{CI}) ; 3$ : for the treatment difference overall, all post-baseline values were included in the model.

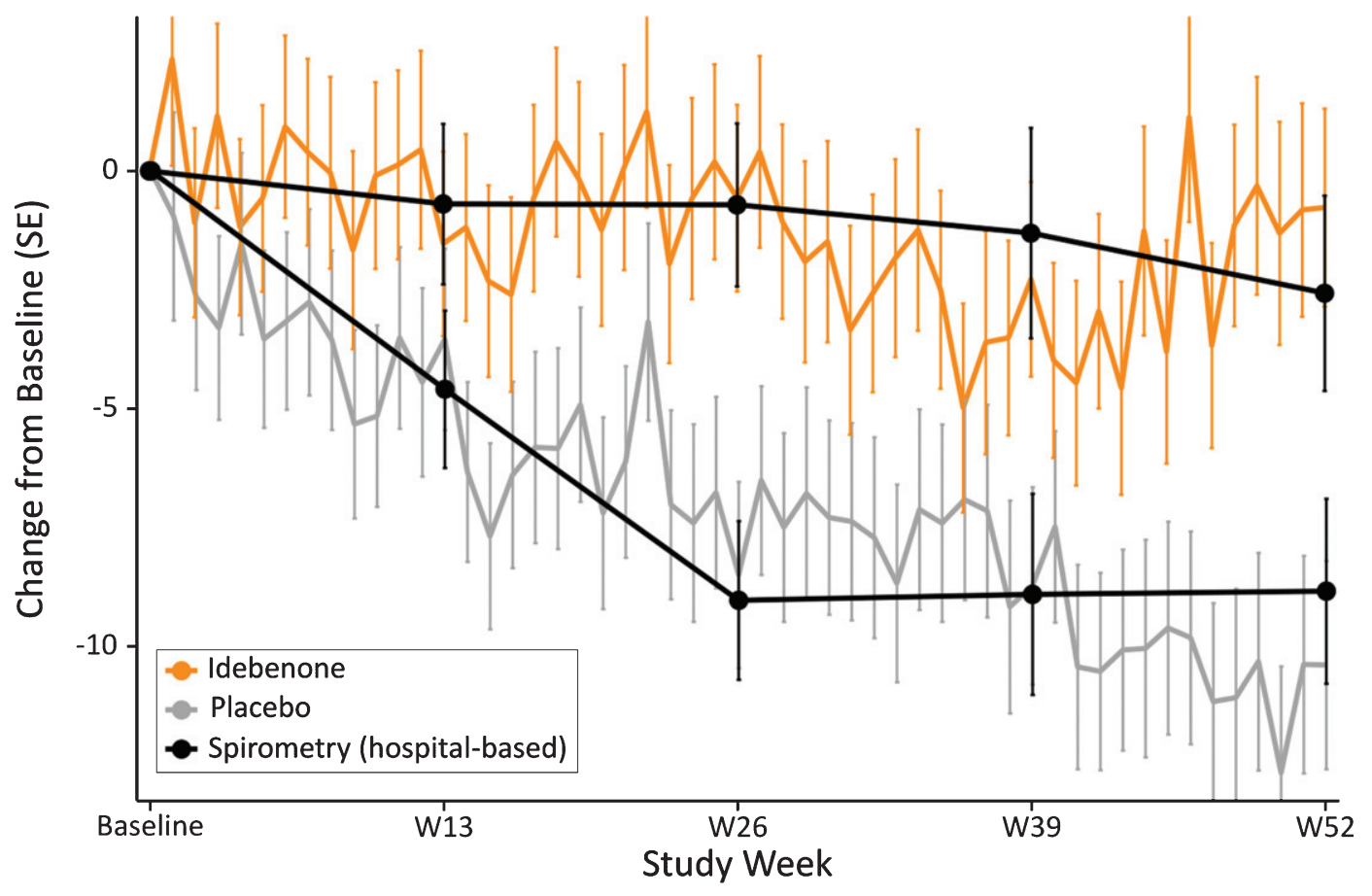

Fig. 7. Change in PEF\%p obtained by weekly HHD compared with hospital-based spirometry results by treatment group.

common pulmonary diseases, such as COPD in adult patients [29].

HHD-based pulmonary function testing also has the potential for being used in the setting of clinical practice monitoring to support patient care. Although regular hospital-based monitoring of pulmonary function is recommended by standard of care guidelines [2, 6, 8-11], compliance is generally poor [21]. Explanations for the limited compliance to regular pulmonary function testing could be that physicians do not recommend testing, or patients are not followed in specialized neuromuscular centres where pulmonary function tests are available and recommended. In this regard it should be emphasized 
that the approach to use HHD-measured pulmonary function data for routine monitoring of disease progression is not limited to the type/brand of device used in this study. In fact, several manufacturers offer suitable devices and provide applications for data analysis and transfer. Certain devices also offer the possibility to collect data for forced vital capacity (FVC), a volume-related pulmonary function measure routinely monitored in patients with DMD. The use of any type of HHD must be accompanied by detailed instructions to the patients and caregivers to ensure reliable pulmonary function measure. This training of patients and caregivers is critical in maximizing the potential for high quality and usable data.

In the current study, patients were advised to use the HHD once per week at home to collect pulmonary function data. However, it could be argued that more frequent (e.g. daily) assessment would offer advantages, particularly an increased number of data points available for interpretation. Moreover, daily use of a HHD would also heighten the awareness for pulmonary aspects of the disease, especially in younger patients where pulmonary function is in the early phase of decline or has not yet reached clinically relevant thresholds. In this regard, daily use of the device could be justified by the short duration (few minutes only) of HHD-based pulmonary testing manoeuvres. However, in patients who already are dependent on the use of cough-assist or ventilator support devices, the additional use of HHD could be seen as extraburden, limiting its acceptance and adherence. For such patients a testing interval of 1 week as studied here should still be acceptable.

In summary, this study demonstrated that homebased pulmonary function testing using a HHD is feasible in DMD patients at age 10-18 years. It should be noted that patients enrolled in the DELOS trial were already in an advanced stage of the disease, as seen in the high proportion of patients who were non-ambulant at baseline and the degree of upper limb weakness [14]. The overall good adherence with the use of the HHD over the course of 1 year indicates that this method could potentially be introduced also for routine monitoring of pulmonary function in the context of patient care. The results shown here also demonstrate that HHDderived data supported the result of the primary endpoint of the DELOS trial, the change to week 52 in PEF\%p, although it is acknowledged that spirometrybased and HHD-based pulmonary function testing outcomes are not independent measures of respiratory function. Specifically, several different analysis methods of HHD-derived data demonstrated a consistent, statistically significant benefit in favour of idebenone in preserving PEF\% $\mathrm{p}$ compared to the placebo group, which followed the expected natural rate of decline [19]. This is the first randomized, controlled clinical trial in DMD in which HHDderived data were pre-planned as an independent validation of the primary, spirometry-based study endpoint.

\section{CONFLICTS OF INTEREST}

GMB, TV, CMMD and OHM are investigators for clinical trials in DMD sponsored by Santhera Pharmaceuticals and act as scientific consultants for Santhera Pharmaceuticals. TV is consultant for Biophytis, Capricor Therapeutics, Italfarmaco, Lysogene, Sarepta, Servier, and Summit. CMMD is consultant for PTC Therapeutics, Biomarin, Sarepta, Eli Lilly, Pfizer, Halo Therapeutics, Cardero Therapeutics, Catabasis, and Mitokyne. OHM has additional consulting relationships with BristolMyers Squib, Capricor Therapeutics, Catabasis, Sarepta, Fibrogen, Biogen, AveXis, and Roche. TM and ML are regular employees of Santhera Pharmaceuticals. CR acts as clinical data scientist for Santhera Pharmaceuticals. GMB and TM are co-inventors of relevant patent applications.

\section{DELOS Study Group (sorted by country)}

Austria: G. Bernert, F. Knipp (Vienna). Belgium: G.M. Buyse, N. Goemans, M. Van den Hauwe (Leuven). France: T. Voit, V. Doppler, T. Gidaro (Paris); J.-M. Cuisset, S. Coopman (Lille). Germany: U. Schara, S. Lutz (Essen); J. Kirschner, S. Borell, M. Will (Freiburg). Italy: M.G. D’Angelo, E. Brighina, S. Gandossini (Lecco); K. Gorni, E. Falcier (Milan); L. Politano, P. D'Ambrosio, A. Taglia (Naples). The Netherlands: J.J.G.M. Verschuuren, C.S.M. Straathof (Leiden). Spain: J.J. Vílchez Padilla, N. Muelas Gómez (Valencia). Sweden: T. Sejersen, M. Hovmöller (Stockholm). Switzerland: P.-Y. Jeannet, C. Bloetzer (Lausanne). USA: S. Iannaccone, D. Castro (Dallas); G. Tennekoon, R. Finkel, C. Bönnemann (Philadelphia); C. McDonald, E. Henricson, N. Joyce (Sacramento); S. Apkon, R.C. Richardson (Seattle). 


\section{ACKNOWLEDGMENTS}

The study was sponsored by Santhera Pharmaceuticals. The DELOS Study Group is indebted to the participating patients and their parents. GMB is Senior Clinical Investigator of the Research Foundation Flanders (FWO Vlaanderen, Belgium).

\section{REFERENCES}

[1] Goemans N, Buyse G. Current treatment and management of Dystrophinopathies. Curr Treat Options Neurol. 2014;16(5).

[2] Bushby K, Finkel R, Birnkrant DJ, et al. Diagnosis and management of Duchenne muscular dystrophy, part 2: Implementation of multidisciplinary care. The Lancet Neurology. 2010;9:177-89.

[3] Passamano L, Taglia A, Palladino A, et al. Improvement of survival in Duchenne muscular dystrophy: Retrospective analysis of 835 patients. Acta Myol. 2012;31:121-25.

[4] Rall S, Grimm T. Survival in Duchenne muscular dystrophy. Acta Myol. 2012;31:117-20.

[5] Schram G, Fournier A, Leduc H, et al. All-cause mortality and cardiovascular outcomes with prophylactic steroid therapy in Duchenne muscular dystrophy. J Am Coll Cardiol. 2013;61:948-54.

[6] Birnkrant DJ, Bushby K, Bann CM, et al. Diagnosis and management of Duchenne muscular dystrophy, part 2: Respiratory, cardiac, bone health, and orthopaedic management. The Lancet Neurology. 2018;17:347-61.

[7] Hollin IL, Peay HL, Apkon SD, Bridges JFP. Patientcentered benefit-risk assessment in duchenne muscular dystrophy. Muscle \& Nerve. 2017;55:626-34.

[8] Birnkrant DJ, Bushby KM, Amin RS, et al. The respiratory management of patients with duchenne muscular dystrophy: A DMD care considerations working group specialty article. Pediatr Pulmonol. 2010;45:739-48.

[9] Finder JD, Birnkrant D, Carl J, et al. Respiratory care of the patient with Duchenne muscular dystrophy: ATS consensus statement. Am J Respir Crit Care Med. 2004;170:456-65.

[10] LoMauro A, D'Angelo MG, Aliverti A. Assessment and management of respiratory function in patients with Duchenne muscular dystrophy: Current and emerging options. Therapeutics and Clinical Risk Management. 2015; 11:1475-88.

[11] Finder J, Mayer OH, Sheehan D, et al. Pulmonary Endpoints in Duchenne Muscular Dystrophy: A Workshop Summary. Am J Respir Crit Care Med. 2017;196:512-9.

[12] Gauld, Gauld LM, Kappers J, et al. Prediction of childhood pulmonary function using ulna length. Am J Respir Crit Care Med. 2003;168:804-9.

[13] Gauld LM, Kappers J, Carlin JB, Robertson CF. Height prediction from ulna length. Dev Med Child Neurol. 2004; 46:475-80.

[14] Meier T, Rummey C, Leinonen M, et al. Characterization of pulmonary function in 10-18 year old patients with Duchenne muscular dystrophy. Neuromuscul Disord. 2017;27:307-14.

[15] McDonald CM, Henricson EK, Abresch RT, et al. The cooperative international neuromuscular research group Duchenne natural history study- a longitudinal investigation in the era of glucocorticoid therapy: Design of protocol and the methods used. Muscle Nerve. 2013;48:32-54.

[16] Mayer OH, Finkel RS, Rummey C, et al. Characterization of pulmonary function in Duchenne muscular dystrophy. Pediatr Pulmonol. 2015;50:487-94.

[17] Mayer OH, Henricson EK, McDonald CM, Buyse GM. Advances in Pulmonary Care in Duchenne Muscular Dystrophy. US Neurology. 2017;13:35-41.

[18] LoMauro A, Romei M, Gandossini S, et al. Evolution of respiratory function in Duchenne muscular dystrophy from childhood to adulthood. Eur Respir J. 2018;51.

[19] McDonald CM, Gordish-Dressman H, Henricson EK, et al. Longitudinal pulmonary function testing outcome measures in Duchenne muscular dystrophy: Long-term natural history with and without glucocorticoids. 2018 Manuscript submitted to Neuromuscular Disorders.

[20] Domingos J, Eagle M, Moraux A, et al. Outcome measures for Duchenne muscular dystrophy from ambulant to non-ambulant patients: Implications for clinical trials. Neuromuscul Disord. 2017;27:S232-S233.

[21] Landfeldt E, Lindgren P, Bell, et al. Compliance to care guidelines for Duchenne muscular dystrophy. J Neuromuscular Diseases. 2015;2:63-72.

[22] Buyse GM, Voit T, Schara U, et al. Efficacy of idebenone on respiratory function in patients with Duchenne muscular dystrophy not using glucocorticoids (DELOS): A doubleblind randomised placebo-controlled phase 3 trial. Lancet. 2015;385:1748-57.

[23] Mayer OH, Leinonen M, Rummey C, et al. Efficacy of idebenone to preserve respiratory function above clinically meaningful thresholds for Forced Vital Capacity (FVC) in patients with Duchenne muscular dystrophy. J Neuromuscular Diseases. 2017;4:189-98.

[24] McDonald CM, Meier T, Voit T, et al. Idebenone reduces respiratory complications in patients with Duchenne muscular dystrophy. Neuromuscul Disord. 2016;26:473-80.

[25] Buyse GM, Voit T, Schara U, et al. Treatment effect of idebenone on inspiratory function in patients with Duchenne muscular dystrophy. Pediatr Pulmonol. 2017;52(4):508-15.

[26] Miller MR. Standardisation of spirometry. Eur Respir J. 2005;26(2):319-38.

[27] Godfrey S, Kamburoff PL, Nairn JR. Spirometry, lung volumes and airway resistance in normal children aged 5 to 18 years. British Journal of Diseases of the Chest. 1970;64: 15-24.

[28] R Core Team. R: A Language and Environment for Statistical Computing. Retrieved from https://www.R-project.org/ (2018)

[29] Rodríguez-Roisin R, Tetzlaff K, Watz H, et al. Daily home-based spirometry during withdrawal of inhaled corticosteroid in severe to very severe chronic obstructive pulmonary disease. Int J COPD. 2016;11:1973-81. 